\title{
Density Estimates of Unmarked Large Mammals at Camera Traps Vary among Models, Species, and Years, Signalling Importance of Model Assumptions
}

\author{
Jason Fisher ${ }^{1}$, Joanna Burgar ${ }^{2}$, Melanie Dickie $^{3}$, Cole Burton ${ }^{4}$, and Rob Serrouya ${ }^{3}$ \\ ${ }^{1}$ University of Victoria \\ ${ }^{2}$ The University of British Columbia \\ ${ }^{3}$ Alberta Biodiversity Monitoring Institute \\ ${ }^{4}$ University of British Columbia
}

November 23, 2021

\begin{abstract}
Density estimation is a key goal in ecology but accurate estimates remain elusive, especially for unmarked animals. Data from camera-trap networks combined with new density estimation models can bridge this gap but recent research has shown marked variability in accuracy, precision, and concordance among estimators. We extend this work by comparing estimates from two different classes of models: unmarked spatial capture-recapture (spatial count, SC) models, and Time In Front of Camera (TIFC) models, a class of random encounter model. We estimated density for four large mammal species with different movement rates, behaviours, and sociality, as these traits directly relate to model assumptions. TIFC density estimates were typically higher than SC model estimates for all species. Black bear TIFC estimates were 10-fold greater than SC estimates. Caribou TIFC estimates were 2-10 fold greater than SC estimates. White-tailed deer TIFC estimates were up to 100-fold greater than SC estimates. Differences of 2-5 fold were common for other species in other years. SC estimates were annually stable except for one social species; TIFC estimates were highly annually variable in some cases and consistent in others. Tests against densities obtained from DNA surveys and aerial surveys also showed variable concordance and divergence. For gregarious animals TIFC may outperform SC due to the latter model's assumption of independent activity centres. For curious animals likely to investigate camera traps, SC may outperform TIFC, which assumes animal behavior is unaffected by cameras. Unmarked models offer great possibilities, but a pragmatic approach employs multiple estimators where possible, considers the ecological plausibility of assumptions, and uses an informed multi-inference approach to seek estimates from models with assumptions best fitting a species' biology.
\end{abstract}

\section{Hosted file}

Density Estimation Ecol Evol Submitted.docx available at https://authorea.com/users/360429/ articles/546600-density-estimates-of-unmarked-large-mammals-at-camera-traps-vary-amongmodels-species-and-years-signalling-importance-of-model-assumptions 